\title{
Perfil cognitivo en pacientes con infección por el virus de inmunodeficiencia humana neurológicamente asintomáticos
}

Cognitive profile in human immunodeficiency virus-infected neurologically asymptomatic patients

\author{
Erik Guevara-Silva ${ }^{1, a}$ \\ Médico Neurólogo, Departamento de Medicina, Hospital Regional de Huacho, Perú. \\ ${ }^{a}$ Maestría en Neurociencias, Universidad Nacional Mayor de San Marcos, Lima, Perú.
}

\begin{abstract}
Resumen
Introducción: Diversos estudios han comunicado la presencia de deterioro cognitivo asociado al virus de inmunodeficiencia humana $(\mathrm{VIH})$ tipo 1, aún en estadios tempranos de la infección. Las principales funciones comprometidas son la memoria a corto plazo, velocidad psicomotriz, atención y función ejecutiva. Objetivos: Describir el perfil cognitivo en pacientes con VIH neurológicamente asintomáticos, antes de iniciar terapia antirretroviral de gran actividad (TARGA), y determinar posibles factores asociados. Diseño: Estudio transversal-analítico. Lugar: Hospital Regional de Huacho. Participantes: Pacientes con VIH y con criterios para ingresar a TARGA. Intervenciones: Veintiún pacientes con VIH, que fueron atendidos desde de abril a julio de 2011, fueron evaluados clínicamente y se les administró una batería neuropsicológica estandarizada. Principales medidas de resultados: Puntajes alcanzados en las pruebas de velocidad psicomotriz, función ejecutiva, memoria inmediata, atención y habilidades visuoespaciales. Resultados: E $47,6 \%$ de pacientes presentó un deterioro cognitivo significativo. Los pacientes fallaron principalmente en las pruebas de velocidad psicomotriz y función ejecutiva. Con excepción de un paciente, todos fracasaron en al menos un test. La edad, tiempo de enfermedad, grado de instrucción y depresión no influyeron significativamente sobre el perfil cognitivo. Conclusiones: Estos hallazgos sugieren que el compromiso cognitivo en algún grado puede presentarse desde las fases tempranas y asintomáticas de la infección por el VIH; estos hallazgos coinciden con la literatura.
\end{abstract}

Palabras clave: Deterioro cognitivo, perfil cognitivo, virus de inmunodeficiencia humana, test neuropsicológico.

Abstract

Background: Several studies have reported cognitive impairment in HIV-1 infected individuals even at early stages of the infection. Short-term memory, psychomotor speed, attention and executive functions are mainly affected. Objectives: To determine the cognitive profile in neurologically asymptomatic HIV-infected patients before starting highly active antiretroviral therapy (HAART), and possible associated factors. Design: Analytic and cross-sectional study. Setting: Hospital Regional de Huacho, Peru. Participants: HIV patients with criteria to start HAART. Interventions: Twenty-one asymptomatic HIV patients attended from April to July 2011 had a detailed clinical history and a battery of standardized neuropsychological tests. Main outcomes measures: Scores in psychomotor speed, executive function, immediate memory, attention, and visuospatial performance evaluations. Results: Significant cognitive impairment was determined in $47.6 \%$ of patients. Patients performed worse in psychomotor speed and executive function tests. All but one patient failed at least in one test. Age, level of instruction, time of disease, and depression were not statistically associated. Conclusions: In accordance with the literature, these findings suggest cognitive decline may occur in early asymptomatic stages of HIV infection.

Key words: Cognitive impairment, cognitive profile, human immunodeficiency virus, neuropsychological test.

An Fac med. 2013;74(1):31-6

\section{INTRODUCCIÓN}

Una de las complicaciones bien conocidas en los pacientes infectados con el virus de inmunodeficiencia humana (VIH) es el deterioro cognitivo, que puede llegar a la demencia, la cual se presenta generalmente en fases avanzadas de la enfermedad, y es de causa multifactorial ${ }^{(1-3)}$. En la década pasada se discutía si este problema se puede presentar tempranamente. $\mathrm{Al}$ respecto varios estudios, aunque en poblaciones pequeñas, han encontrado que el dete- rioro cognitivo se puede presentar en pacientes con VIH asintomáticos ${ }^{(4,5)}$. Además, la falta de anormalidades cognitivas encontrada mediante estudios transversales no excluye la posibilidad que existan algunos cambios en el aspecto cognitivo en las fases iniciales o estadio asintomático de la infección ${ }^{(6)}$. Muchos de estos estudios fueron realizados antes de la introducción de la terapia antirretroviral de gran actividad (TARGA), época en la cual el tiempo promedio de muerte en los pacientes con diferentes grados de severidad del complejo demencia sida era aproximadamente 6 meses ${ }^{(7)}$.

La disfunción cognitiva en los pacientes con VIH está asociada a la cantidad de virus en sangre y al ingreso al sistema nervioso desde los primeros días de la infección. Esta disfunción puede llegar a la demencia progresiva, caracterizada por cambios motores, cognitivos y conductuales. Comparado con grupos controles, estos pacientes también pueden presentar anormalidades cognitivas menos severas demostradas 
Tabla 1. Clasificación de los signos y sintomas de la encefalitis por VIH ${ }^{(14)}$.

\begin{tabular}{|l|l|}
\hline Hallazgos neurológicos & \multicolumn{2}{|c|}{ Signos de la encefalitis por VIH } \\
& $\begin{array}{l}\text { Fase temprana: trastorno de la marcha, lentitud motora, hipomimia, tremor. } \\
\text { esfinteriano. Reflejos palmomentoniano, glabelar y de prehensión. } \\
\text { Fase terminal: cuadriplejia espástica y compromiso de ambos esfínteres. }\end{array}$ \\
\hline Hallazgos neuropsicológicos & Lentitud psicomotora, deterioro de memoria a corto plazo. \\
\hline Hallazgos psicológicos & $\begin{array}{l}\text { Fase temprana: aplanamiento emocional, falta de iniciativa, distraibilidad. } \\
\text { Fase tardía: problemas para recordar eventos en el correcto orden temporal, desorientación } \\
\text { en tiempo y espacio. Finalmente la presencia de mutismo. }\end{array}$ \\
\hline
\end{tabular}

por evaluaciones neuropsicológicas; a esto se ha denominado disfunción cognitivo-motora mínima ${ }^{(8)}$. Sin embargo, existen otros factores, como patologías psiquiátricas, drogas y el abuso de alcohol, que parecen afectar las capacidades cognitivas en mayor grado que el VIH ${ }^{(9)}$. Dentro de las funciones cognitivas principalmente comprometidas en esta población tenemos a la velocidad psicomotora, memoria, función ejecutiva, atención y habilidades visuoespaciales ${ }^{(10-13)}$.

Las complicaciones neurológicas están íntimamente relacionadas al tiempo de infección. Dentro de las manifestaciones producidas por invasión del VIH al sistema nervioso central están la encefalopatía por VIH, que puede ir de un deterioro cognitivo leve hasta una demencia de tipo subcortical (complejo demencia-sida), y la presencia de síntomas neuropsiquiátricos. Estos síntomas pueden ser agrupados como se muestra en la tabla $1^{(14) .}$ La severidad del complejo demencia-sida puede ser clasificada de acuerdo a los criterios de la esca- la del Memorial Sloan Kettering ${ }^{(15)}$, la cual en esencia viene a ser una escala de Karnofsky modificada (tabla 2).

El Centro para el Control y Prevención de Enfermedades (CDC) en Estados Unidos ha clasificado a los pacientes con VIH en base a criterios clínicos y el conteo de linfocitos T-CD4 para precisar mejor la progresión y la morbilidad relacionada al VIH y guiar el tratamiento antirretroviral ${ }^{(16)}$.

Existen pruebas cognitivas estandarizadas y ampliamente utilizadas recomendadas por la OMS ${ }^{(13,17)}$, muchas de ellas validadas al idioma castellano, para evaluar el perfil cognitivo de los pacientes con VIH ${ }^{(12,18-20)}$. Algunas de estas pruebas son tomadas del test conocidos en nuestro medio como la $\mathrm{Ba}$ tería Neuropsicológica Breve (Neuropsi) y el Wechsler Adult Intelligence Scale (WAIS) ${ }^{(21,22) .}$

El objetivo de la presente investigación fue describir el componente cognitivo de un grupo de pacientes neurológicamente asintomáticos con infección por VIH, pero con compromiso del sistema inmune suficiente para el inicio de TARGA, y determinar posibles factores asociados.

\section{MÉTODOS}

Esta investigación ha sido aprobada por la dirección general del Hospital Regional de Huacho. Todos los pacientes aceptaron su participación por escrito. Se realizó un análisis transversal de la evaluación cognitiva de los pacientes adultos con VIH que acudieron a PROCETSS entre los meses de abril a julio de 2011 (seleccionados de forma no aleatoria), pertenecientes al estadio A o B de la clasificación de la CDC. Estos pacientes recibieron un examen neurológico exhaustivo por parte del investigador. Cumplían los criterios inmunológicos (células T CD4 menor a $200 \mathrm{cel} / \mathrm{mL}$ ) o virológicos (carga viral mayor a 55000 copias $/ \mathrm{mL}$ o su logaritmo 4,74), según el Ministerio de Salud ${ }^{(23)}$, para iniciar TARGA. Fueron excluidos los pacientes con anteceden-

Tabla 2. Estadios del complejo demencia-sida.

\begin{tabular}{l|l}
\hline Estadio 0 & $\begin{array}{l}\text { Función mental y motora normales. } \\
\text { Estadio } 0,5 \text { (subclínico) }\end{array}$ \\
Estadio 1 (leve) & $\begin{array}{l}\text { Déficit cognitivo que compromete la realización de los aspectos más demandantes del trabajo o de las } \\
\text { actividades de la vida diaria. Puede caminar sin ayuda. }\end{array}$ \\
Estadio 2 (moderado) & $\begin{array}{l}\text { Déficit cognitivo que impide al paciente trabajar o realizar las actividades de la vida diaria más importantes. } \\
\text { Déficit cognitivo que solo permite realizar tareas muy básicas; se requiere asistencia para caminar. }\end{array}$ \\
Estadio 3 (severo) & $\begin{array}{l}\text { El paciente no comprende lo que ocurre a su alrededor, mutismo. Paraparesia con compromiso esfinteriano } \\
\text { doble. }\end{array}$ \\
\hline
\end{tabular}


tes de enfermedades neurológicas o psiquiátricas, abuso de alcohol o drogas, consumo de fármacos (benzodiacepinas, antidepresivos, neurolépticos), estar en TARGA o haberlo recibido anteriormente. Los datos epidemiológicos (edad, sexo, educación, procedencia) y clínicos (tiempo de infección por $\mathrm{VIH}$, enfermedades previas) y conteo de CD4 en el momento de la evaluación neuropsicológica, fueron recolectados directamente de los pacientes o tomados de las historias clínicas; luego fueron anotados en formatos estandarizados. El tiempo de infección por el VIH fue determinado en base a la fecha del examen de ELISA positivo confirmado por western blot, que figuraba en las historias clínicas.

La función cognitiva es una variable multidimensional, la cual ha sido dividida en seis dimensiones para su mejor análisis: función ejecutiva, velocidad psicomotriz, memoria visual inmediata, memoria auditiva inmediata, atención y habilidad visuoespacial. Estas fueron evaluadas mediante el test del Trazo A (trail making test, TMT), test del Trazo B, codificación de dígitos y símbolos, test de aprendizaje audioverbal de Rey, prueba de retención de dígitos y dibujo de una figura semicompleja, respectivamente. Algunas de estas pruebas han sido tomadas del WAIS y Neuropsi, y todas ellas han sido ampliamente utilizadas en la evaluación de pacientes con $\mathrm{VIH}$ en publicaciones previas, $\mathrm{y}$ validadas al idioma castellano. Los puntajes alcanzados por cada paciente fueron estandarizados utilizando medias ajustadas según datos demográficos (edad, grado de instrucción) ${ }^{(18,21,22)}$. Además, se aplicó la escala de Beck para determinar la presencia de síntomas depresivos.

En el análisis estadístico, la variable dependiente (función cognitiva) fue medida como una variable de tipo semicontinua (expresada por el puntaje o valor absoluto del resultado de cada una de las seis pruebas). Así, cada uno de los individuos de estudio tuvo un set de seis resultados. Para resumir cada uno de estos seis resultados (velo- cidad psicomotora, atención, memoria inmediata visual, memoria inmediata auditiva, función ejecutiva y habilidad visuoespacial), se utilizó medidas de tendencia central (media y mediana) así como de dispersión (desviación estándar y rango intercuartílico). Este indicador resume lo que sucede en la población de estudio por cada una de las seis dimensiones de estudio. Las variables categóricas fueron resumidas usando frecuencias absolutas y relativas, y las variables cuantitativas mediante el uso de medidas de tendencia central (media y mediana), así como de dispersión (desviación estándar y rango intercuartílico). Se aplicó la prueba de correlación de Pearson para buscar asociaciones con las variables edad, tiempo de enfermedad y grado de instrucción. Se creó una nueva categoría 'deterioro cognitivo', definida como el fracaso en tres o más de las pruebas aplicadas; esta variable fue comparada con la presencia de depresión, sexo, edad y tiempo de enfermedad, mediante las pruebas no paramétricas de chi cuadrado o Wilcoxon, por tratarse de una muestra no aleatoria. Se consideró un valor de $\mathrm{p}<$ 0,05 como criterio para establecer una significancia estadística. Los análisis fueron ejecutados utilizando el paquete estadístico SPSS para Windows versión 18.

\section{RESULTADOS}

Este trabajo descriptivo incluyó a 21 pacientes, 16 varones y 5 mujeres. La edad promedio fue 33 años; la mayoría había completado los estudios primarios y el tiempo de enfermedad varió de 1 a 240 meses (tabla 3). El promedio de

Tabla 3. Características epidemiológicas de los pacientes con VIH.

\begin{tabular}{|c|c|c|c|}
\hline Variable & & Número & Porcentaje \\
\hline Sexo & $\begin{array}{l}\text { Varones } \\
\text { Mujeres }\end{array}$ & $\begin{array}{l}16 \\
5\end{array}$ & $\begin{array}{l}76,1 \\
23,9\end{array}$ \\
\hline Años de estudio* & $\begin{array}{c}0 \text { a } 5 \\
6 \text { a } 11 \\
\text { Más de } 11\end{array}$ & $\begin{array}{c}2 \\
11 \\
8 \\
\end{array}$ & $\begin{array}{c}9,5 \\
52,3 \\
38,2 \\
\end{array}$ \\
\hline Edad & $\begin{array}{c}\text { Promedio (DE) } \\
01 \\
02 \\
03 \\
\end{array}$ & $\begin{array}{c}33,1(7,0) \\
28 \\
32 \\
35\end{array}$ & - \\
\hline $\begin{array}{c}\text { Tiempo de infección } \\
\text { por el VIH }\end{array}$ & $\begin{array}{c}\text { Promedio (DE) } \\
01 \\
02 \\
03\end{array}$ & $\begin{array}{c}38,5(60,1) \\
2 \\
8 \\
52 \\
\end{array}$ & - \\
\hline
\end{tabular}

* Indica el grado de instrucción de cada paciente medido en años culminados durante el colegio y estudios técnicos y/o universitarios.

DE: desviación estándar. Q1: primer cuartil. Q2: segundo cuartil. Q3: tercer cuartil.

Tabla 4. Puntaje alcanzado en la evaluación de las funciones cognitivas de los pacientes con VIH.

\begin{tabular}{|c|c|c|c|c|}
\hline Función cognitiva & Media & DE* & Mediana & IQR $^{* *}$ \\
\hline Función ejecutiva & 76,5 & 25,1 & 77 & 36 \\
\hline Velocidad psicomotriz & 165,6 & 78,3 & 144 & 135 \\
\hline Memoria inmediata visual & 66,1 & 11,8 & 70 & 21 \\
\hline Memoria inmediata verbal & 12,7 & 2,4 & 14 & 4 \\
\hline Atención & 13 & 2,4 & 14 & 5 \\
\hline Habilidad visuoespacial & 11,9 & 0,2 & 12 & 0 \\
\hline
\end{tabular}

* DE: desviación estándar. ** $\mathrm{QR}$ : rango intercuartil. 
Tabla 5. Número de pacientes que fracasaron en las pruebas de evaluación cognitiva.

\begin{tabular}{llcc} 
Función ejecutiva & Herramienta & $\begin{array}{c}\text { No pacientes que } \\
\text { fallaron el test }\end{array}$ & $\%$ \\
1. Función ejecutiva & Test del Trazo A & 15 & 71 \\
2. Velocidad psicomotriz & Test del Trazo B & 16 & 76 \\
3. Memoria visual inmediata & Codificación de símbolos y dígitos & 8 & 38 \\
4. Memoria verbal inmediata & Test audioverbal de Rey & 3 & 14,2 \\
5. Atención & Retención de dígitos & 10 & 47,6 \\
6. Habilidad visuoespacial & Dibujo de figura & 1 & 4,7 \\
\hline
\end{tabular}

CD4 fue $147,3(\mathrm{DE}=122)$ y de carga viral ( $\log 10), 4,9(\mathrm{DE}=1,1)$. Ningún paciente indicó síntomas neurológicos. Según la evaluación neurológica somática, ningún paciente presentó signos de compromiso del sistema nervioso central o periférico. Sin embargo, en la evaluación de las funciones superiores, en base a las pruebas mencionadas anteriormente, se encontró resultados diversos que son detallados en la tabla 4. En las pruebas de memoria inmediata, atención y habilidad visuoespacial, los puntajes más altos corresponden a un mejor rendimiento de la prueba; por el contrario, en las pruebas de función ejecutiva y velocidad psicomotriz, los puntajes más elevados (tiempo en segundos para ejecutar el test) corresponden a un menor rendimiento.

Se encontró que la mayoría de errores fue cometida a la hora de ejecutar las pruebas de función ejecutiva y velocidad psicomotriz (tabla 5). De los 21 pacientes evaluados, solo un paciente realizó correctamente todas las evaluaciones, tres pacientes fracasaron en al menos una prueba, 7 fracasaron en al menos dos pruebas, 5 fracasaron en al menos 3 pruebas, 3 pacientes fracasaron en al menos 4 pruebas y 2 pacientes fracasaron en al menos 5 de las 6 pruebas neuropsicológicas. Diez pacientes $(47,6 \%)$ presentaron un deterioro cognitivo significativo (fracaso en tres o más pruebas neuropsicológicas).

No existió asociación significativa cuando se evaluó los puntajes de cada test en función de la edad, tiempo de infección y grado de instrucción ( $p$
$>0,05)$. La presencia de síntomas depresivos no afectó significativamente la presencia de deterioro cognitivo $(\mathrm{p}=$ 0,1 , mediante la prueba de chi al cuadrado).

\section{DISCUSIÓN}

Las manifestaciones neuropsiquiátricas debidas a la infección del virus de inmunodeficiencia humana han sido redescripciones sobre el VIH, en 1981, y pueden iniciarse incluso como un detenas y en personas por lo demás asintomáticas, cuando se utiliza evaluaciones neuropsicológicas apropiadas ${ }^{(4,5,24)}$. De acuerdo a esto, nosotros hemos encontrado algún grado de déficit cognitivo en todos excepto uno de los 21 pacientes evaluados, y 10 pacientes mostraron un deterioro cognitivo significativo, pero sin llegar al grado de demencia. Sin embargo, no todos los estudios muestran los mismos resultados. Selnes siguió por un año y medio a una cohorte de 238 pacientes con sus respectivos controles, sin encontrar que el deterioro gradual cognitivo ocurriera en las fases asintomáticas de la infección por $\mathrm{VIH}^{(25)}$; lo mismo ocurrió con otros dos estudios realizados en Italia, en 328 y 165 pacientes ${ }^{(6,26)}$. Esta disparidad de resultados podría deberse a factores distintos al VIH, como el consumo de drogas ${ }^{(27)}$ o el estado de ánimo ${ }^{(28)}$, grado de instrucción y la reserva cognitiva como factores protectores ${ }^{(29)}$, y también a la conocidas cada vez con mayor frecuencia desde que aparecieron las primeras rioro cognitivo leve en las fases tempra- presencia de factores genéticos ${ }^{(30)}$. En este estudio, no se evidenció que la presencia de síntomas depresivos, edad, el grado de instrucción o tiempo de enfermedad influyeran significativamente en el rendimiento en las pruebas neuropsicológicas, probablemente debido a que la muestra fue pequeña. A diferencia de los trabajos mencionados, nosotros evaluamos solo a aquellos pacientes asintomáticos que ya habían sido seleccionados para recibir TARGA; es decir, presentaban un compromiso inmunológico y /o virológico significativo; esto podría explicar en parte el compromiso cognitivo encontrado en la mayoría de ellos.

El compromiso cognitivo es más evidente en las áreas de velocidad psicomotriz, resolución de problemas y habilidades visuoespaciales, los cuales más adelante van a caracterizar a una demencia de tipo subcortical (1). El deterioro cognitivo en estadios tempranos de la infección también ha sido encontrado ampliamente por diversos investigadores ${ }^{(3-6)}$, utilizando pruebas como el test del Trazo A y B (función ejecutiva y velocidad psicomotriz) y algunos subtests tomados del WAIS; es decir, con las mismas herramientas utilizadas en este estudio, validadas al idioma castellano. En esta investigación se describe el perfil cognitivo de 21 pacientes neurológicamente asintomáticos al momento del estudio. Todos ellos recibieron una batería de pruebas neuropsicológicas, encontrándose que la mayoría fracasaba en las pruebas de función ejecutiva y velocidad psicomotriz, principalmente, así como también se evidenció afectación de la memoria visual inmediata y atención.

La mayor información sobre VIH y compromiso cognitivo se obtiene de estudios extranjeros; sin embargo, la diversidad cultural y la diferencia de idiomas hace difícil poder extrapolar estos resultados. En consecuencia, se hace necesario diseñar estudios en nuestras poblaciones con herramientas de evaluación cognitiva validadas al español. Considerando que el idioma español es la primera lengua en casi el 10\% de la 
población mundial, un grupo de investigadores validaron el Neuropsi en una población mexicana ${ }^{(21)}$ y otro grupo de expertos crearon el HUMANS para pacientes con VIH de habla castellana ${ }^{(19)}$. Por otro lado, la elevada incidencia y prevalencia de VIH en América Latina y particularmente en el Perú, donde tenemos 28948 casos de sida y 46749 casos de infección por VIH, notificados hasta abril de $2012^{(31)}$, nos obliga a los neurólogos conocer y realizar investigaciones en nuestra propia población para contrastarla con otros resultados y poder plantear medidas preventivas y terapéuticas oportunas. Sin embargo, es poco lo que se ha escrito en relación al compromiso cognitivo en estos pacientes ${ }^{(32)}$. En la ciudad de Huacho existe una importante población de pacientes con VIH que asisten a EsSalud o al hospital Regional de Huacho (entre 4 a 6 pacientes ingresan a TARGA cada mes), pero en ninguno de ellos se ha realizado investigaciones en relación a este tema.

En esta investigación se puede encontrar algunas limitaciones propias de un estudio descriptivo transversal; la falta de un grupo control, así como el tamaño de la muestra y la selección no aleatoria de la misma. Sin embargo, se puede evidenciar que el compromiso cognitivo se presenta en pacientes neurológicamente asintomáticos y esto justificaría que todos los pacientes sean evaluados en consultorio de neurología antes de iniciar TARGA, tal como lo recomienda el Ministerio de Salud ${ }^{(23)}$. La detección temprana de este problema es importante para conocer mejor el curso de la enfermedad, ofrecer tempranamente terapias cognitivas-conductuales y tener potenciales candidatos para ensayos con futuros fármacos dirigidos a tratar el deterioro cognitivo asociado al VIH.

Básicamente, los resultados de este estudio nos demuestran la existencia de deterioro de las funciones cognitivas en pacientes sin sintomatología neurológica evidente. Estos resultados coinciden con lo encontrado por otros autores en la literatura extranjera; sin embargo, el tamaño de la población no nos permite hacer conclusiones respecto a la asociación con otros factores de riesgo. Todo paciente debe recibir una evaluación neuropsicológica antes de iniciar TARGA para conocer el estado basal del perfil cognitivo y determinar, mediante posteriores evaluaciones, en qué momento estas capacidades empiezan a declinar. Estos resultados deben ser corroborados por otras investigaciones nacionales en poblaciones más grandes y con grupos control. Se hace necesaria la estandarización de una batería neuropsicológica en pacientes con VIH en el Perú.

\section{AGRADECIMIENTOS}

Dr. Miguel Angel Estela. Infectólogo del Hospital Regional de Huacho.

Lic. Nila Pajuelo. Psicóloga del Hospital Regional de Huacho.

Dr. Eddy Segura. Epidemiólogo, Universidad Peruana Cayetano Heredia.

\section{REFERENCIAS BIBLIOGRÁFICAS}

1. Tross S, Price RW, Navia B, Thaler HT, Gold J, Hirsch DA, et al. Neuropsychological characterization of the AIDS dementia complex: a preliminary report. AIDS. 2008;2:81-88.

2. McArthur JC, McClernon DR, Cronin MF, NanceSproson TE, Saah AJ, St Clair M, et al. Relationship between human immunodeficiency virus-associated dementia and viral load in cerebrospinal fluid and brain. Ann Neurol. 1997;42:689-98.

3. Stern Y, Liu X, Marder K, Todak G, Sano M, Ehrhardt $A$, et al. Neuropsychological changes in a prospectively followed cohort of homosexual and bisexual men with and without HIV infection. Neurology. 1987;45:467-72.

4. Grant I, Atkinson JH, Hesselink JR, Kennedy CJ, Richman DD, Spector SA, et al. Evidence for early central nervous system involvement in the acquired immunodeficiency syndrome (AIDS) and other human immunodeficiency virus (HIV) infections. Ann Int Med. 1987;107:828-36.

5. Poutiainen E, Livanainen M, Elovaara I, Valle S-L, Landevirta J. Cognitive changes as early signs of HIV infection. Acta Neurol Scand. 1988;78:49-52.

6. Grassi M, Perin C, Borella M, Mangoni A. Assessment of cognitive function in asymptomatic HIVpositive subjects. Eur Neurol. 1999;42:225-9.

7. Bouwman F, Skolasky R, Hes D, Selnes O, Glass J, Nance-Sproson T, et al. Variable progression of HIV-associated dementia. Neurology. 1998;50:1814-20.

8. Working Group of the American Academy of Neurology AIDS Task Force. Nomenclature and research case definitions for neurologic manifestations of human immunodeficiency virus-type 1 (HIV-1) infection. Neurology. 1991;41:778-85.

9. Vázquez-Justo, E., Rodríguez-Álvarez, M. Influencia de factores no relacionados con la infección en el rendimiento neuropsicológico de seropositivos al VIH. Rev Neurol. 2002;35:474-80.

10. Sacktor N, Tarwater P, Skolasky R, McArthur JC, Selnes OA, Beckeret J, et al. CSF antiretroviral drug penetrance and the treatment of HIV-associated psychomotor slowing. Neurology. 2001;57:542-4.

11. Marra CM, Lockhart D, Zunt JR, Perrin M, Coombs RW, Collier AC. Changes in CSF and plasma HIV-1 RNA and cognition after starting potent antiretroviral therapy. Neurology. 2003;60:1388-90.

12. Butters N, Grant I, Haxby J, Judd LL, Martin A, McClelland J, et al. Assessment of Aids-related cognitive changes: Recommendations of the NIMH Workshop on neuropsychological assessment approaches. J Clin Exp Neuropsychol. 1990;12:963-78.

13. Maj M, Starace F, Sartorius N. Neuropsychiatric aspects of HIV-1 infection: data collection instrument for a WHO cross-cultural study. WHO Bull. 1991;69:243-5.

14. Hoffman C, Rockstroh J, Kamps B. HIV Medicine. $14^{\mathrm{a}}$ ed. Paris: Flying Publisher. 2006:646-7.

15. Price RW, Siditis JJ. Evaluation of the AIDS dementia complex in clinical trials. J AIDS. 1990;3(Suppl 2):S51-S60.

16. Centers for Disease Control and Prevention: 1993 revised classification system for HIV infection and expanded surveillance case definition for AIDS among adolescents and adult. MMWR. 1992;41:1-4.

17. Maj M, Janssen R, Satz P, Zaudig M, Starace F, Boor D, et al. The world health Organization's cross-cultural study on neuropsychiatric aspects of infection with the human inmunodeficiency virus 1 (HIV-1). Preparation and pilot phase. $\mathrm{Br} \mathrm{J}$ Psychiatry. 1991;159:351-6.

18. Gonzáles-Hernandez, J. Bateria neuropsicológica "memoriza". Tomado el 15 de Noviembre, 2010, de la Pontificia Universidad Católica de Chile, Departamento de Neurologia. Página web: http:// www.memoriza.com

19. Ardila-Ardila, A, Goodkin K, Concha-Bartolini M, Lecusay-Ruiz R, O'Mellan-Fajardo S, Suárez-Bustamante $P$, et al. HUMANS: una bateria neuropsicológica para la evaluación de pacientes infectados con VIH-1. Rev Neurol. 2003;36:756-62.

20. Martínez C, Ramírez L, De la Rosa N. Complejo demencial asociado a VIH/SIDA: deterioro cognitivo asociado a la evolución de una enfermedad. VI Congreso Nacional sobre SIDA 1997. Biblioteca virtual VIH/SIDA. http://bvssida.insp.mx/harticulo. php?id_art=1009\&seltabla $=1$

21. Ostrosky-Solis F, Ardila A, Rosselli M. NEUROPSI evaluación neuropsicológica breve en español: manual e instructivo. México: Publingenio. 1991:1-8.

22. Marta, H. La Escala de Inteligencia de Wechsler para adultos (WAIS): Manual. Chile: Pontificia Universidad Católica de Chile, Escuela de Psicología. 1982:3-63.

23. Ministerio de Salud. Dirección General de Salud de las Personas. Norma técnica para el tratamiento antiretroviral de gran actividad - TARGA en adultos infectados por el virus de la inmunodeficiencia humana. Perú. 2004:4-6.

24. White D, Heaton R, Monsch A. Neuropsychological studies of asymptomatic human inmunodeficiency virus-type-1 infected individuals. J Int Neuropsychol Soc. 1995;1:304-15. 
25. Selnes O, Miller E, McArthur J. HIV-1 infection: No evidence of cognitive decline during the asymptomatic stages. Neurology. 1990;40:204-8.

26. Giancola M, Lorenzini P, Balestra P, Larussa D, Baldini F, Corpolongo A, et al. Neuroactive antiretroviral drugs do not influence neurocognitive performance in less advanced HIV-infected patients responding to HAART. JAIDS. 2006;41:332-7.

27. Reed RJ, Grant J. The long-term neurobehavioral consequences of substance abuse: conceptual and methodological challenges for future research. NIDA Res Monogr. 1990;101:1056.

28. Bornstein RA, Pace $P$, Rosenberger $P$, Nasrallah HA, Para MF, Whitacre CC, et al. Depression and neuropsychological performance in asymptomatic HIV infection. Am J Psychiatry. 1993;150:922-7.
29. Pereda M, Ayuso-Mateos J L, del Barrio AG, Echevarria S, Farinas MC, Palomo D, et al. Factors associated with neuropsychological performance in HIVseropositive subjects without AIDS. Psychol Med. 2000;30:205-17.

30. Gonzalez E, Rovin BH, Sen L, Cooke G, Dhanda R, Mummidi S, et al. HIV-1 infection and AIDS dementia are influenced by a mutant MCP-1 allele linked to increased monocyte infiltration of tissues and MCP-1 levels. Proc Natl Acad Sci USA. 2002;99:13795-800.

31. Ministerio de Salud. Boletín VIH SIDA, Epidemiología. Casos de SIDA VIH por Año de Diagnóstico al 30 Abril 2012. http://www.app.minsa.gob.pe/bsc/ detalle_indbsc.asp? $|c i n d=18 \&| c o b j=4 \& \mid c p e r=1 \&$ Icfreg $=22 / 5 / 2012$
32. Custodio N, Escobar J, Altamirano J. Demencia asociada a infección por virus de inmunodeficiencia humana tipo 1. An Fac med. 2006;67:243-54.

Artículo recibido el 2 de agosto de 2012 y aceptado para publicación el 10 de octubre de 2012.

Correspondencia:

Dr. Erik Guevara Silva

Teléfono: (511) 998895959 - Telefax: (511) 5680279

Correo electrónico: erikguevara@hotmail.com 